\title{
Photo-enhanced magnetization in Fe-doped ZnO nanowires
}

I. Lorite, Y. Kumar, P. Esquinazi, S. Friedländer, A. Pöppl, T. Michalsky, J. Meijer, M. Grundmann, T. Meyer, and I. Estrela-Lopis

Citation: Appl. Phys. Lett. 109, 012401 (2016); doi: 10.1063/1.4955048

View online: https://doi.org/10.1063/1.4955048

View Table of Contents: http://aip.scitation.org/toc/apl/109/1

Published by the American Institute of Physics

\section{Articles you may be interested in}

A comprehensive review of $\mathrm{ZnO}$ materials and devices

Journal of Applied Physics 98, 041301 (2005); 10.1063/1.1992666

Ultraviolet electroluminescence from $\mathrm{Au}-\mathrm{ZnO}$ nanowire Schottky type light-emitting diodes

Applied Physics Letters 108, 261103 (2016); 10.1063/1.4954758

Carrier relaxation in (In, Ga)As quantum dots with magnetic field-induced anharmonic level structure Applied Physics Letters 109, 012103 (2016); 10.1063/1.4955459

Confinement-driven metal-insulator transition and polarity-controlled conductivity of epitaxial $\mathrm{LaNiO}_{3} / \mathrm{LaAlO}_{3}$ (111) superlattices

Applied Physics Letters 109, 082108 (2016); 10.1063/1.4961693

Robust ferromagnetism in Mn-doped $\mathrm{MoS}_{2}$ nanostructures

Applied Physics Letters 109, 092401 (2016); 10.1063/1.4961883

Advances in methods to obtain and characterise room temperature magnetic $\mathrm{ZnO}$

Applied Physics Letters 106, 082406 (2015); 10.1063/1.4913763

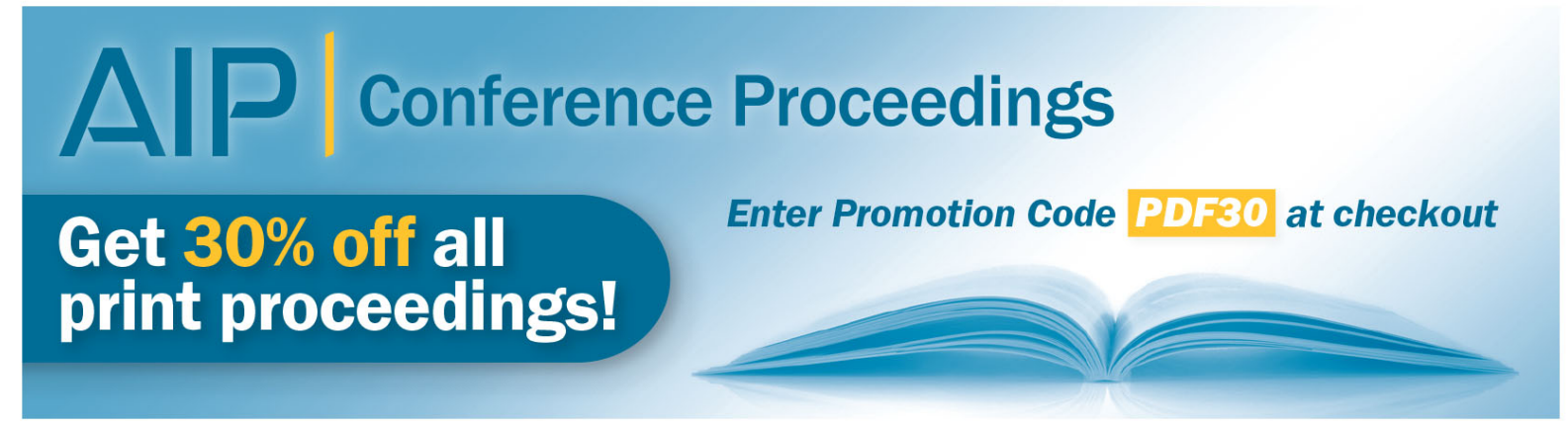




\title{
Photo-enhanced magnetization in Fe-doped $\mathrm{ZnO}$ nanowires
}

\author{
I. Lorite, ${ }^{1, a)}$ Y. Kumar, ${ }^{1}$ P. Esquinazi, ${ }^{1}$ S. Friedländer, ${ }^{1}$ A. Pöppl, ${ }^{1}$ T. Michalsky, ${ }^{1}$ J. Meijer, ${ }^{1}$ \\ M. Grundmann, T. Meyer, ${ }^{2}$ and I. Estrela-Lopis ${ }^{2}$ \\ ${ }^{1}$ Institut für Experimentelle Physik II, University of Leipzig, Linnéstraße 5, D-04103 Leipzig, Germany \\ ${ }^{2}$ Institut für Medizinische Physik und Biophysik, University of Leipzig, Härtelstraße 16-18, D-04107 Leipzig, \\ Germany
}

(Received 3 May 2016; accepted 19 June 2016; published online 6 July 2016)

\begin{abstract}
An emerging branch of electronics, the optospintronics, would be highly boosted if the control of magnetic order by light is implemented in magnetic semiconductors' nanostructures being compatible with the actual technology. Here, we show that the ferromagnetic magnetization of low Fe-doped $\mathrm{ZnO}$ nanowires prepared by carbothermal process is enhanced under illumination up to temperatures slightly below room temperature. This enhancement is related to the existence of an oxygen vacancy $\mathrm{V}_{\mathrm{O}}$ in the neighborhood of an antiferromagnetic superexchange $\mathrm{Fe}^{3+}-\mathrm{Fe}^{3+}$ pair. Under illumination, the $\mathrm{V}_{\mathrm{O}}$ is ionized to $\mathrm{V}_{\mathrm{O}}^{+}$giving an electron to a close $\mathrm{Fe}^{3+}$ ion from the antiferromagnetic pair. This light excited electron transition allows the transition of $\mathrm{Fe}^{3+}$ to $\mathrm{Fe}^{2+}$ forming stable ferromagnetic double exchange pairs, increasing the total magnetization. The results presented here indicate an efficient way to influence the magnetic properties of $\mathrm{ZnO}$ based nanostructures by light illumination at high temperatures. Published by AIP Publishing.
\end{abstract}

[http://dx.doi.org/10.1063/1.4955048]

Magnetic materials with addressable electrical and/or optical properties may add flexibility and larger storage density to spintronics devices. The photo-induced magnetization (PIM) of materials is of interest because it would lead to the development of optospintronic devices. The PIM phenomenon has been found below a temperature of $T \simeq 19 \mathrm{~K}$ in cobalt-iron cyanide-based Prussian blue analog, ${ }^{1} T \simeq 35 \mathrm{~K}$ for magnetic semiconductor heterostructures, ${ }^{2}$ and $T \simeq 80 \mathrm{~K}$ for core-shell nanostructures. ${ }^{3}$ Recently, PIM after irradiation of polarized light in oxygen-deficient $\mathrm{SrTiO}_{3}$ and at $T<10 \mathrm{~K}$ has been reported, ${ }^{4}$ revealing the importance of certain defects for a possible manipulation of magnetization by light. The photo induced magnetization has also been observed at room temperature, RT, in non-epitaxial film with a nano-crystallite size of $17 \mathrm{~nm} .^{5}$ The results in Ref. 5 suggested that a stronger spin-orbit interaction given by small crystallite size samples is essential to generate the PIM in manganese zinc ferrite films. ${ }^{5}$ However, it hinders the possible applications in spintronic since small spin-orbit interaction is ideally required for a larger spin coherence length. In particular, this is the case of $\mathrm{ZnO}$ based defect induced magnetic (DIM) materials, which are ideal candidates not only due to the large spin coherence length, ${ }^{6}$ but also because of the observed variation in the optoelectronic properties at low magnetic fields, i.e., $0.4 \mathrm{~T}^{7}$ In fact, studies have already been performed in colloidal nanocrystals of $\mathrm{Mn}^{2+}$ doped $\mathrm{ZnO}$ showing a PIM property but only below $T \sim 2 \mathrm{~K}$ as shown by magnetization measurements. ${ }^{8}$ It is, therefore, of interest to further study PIM in magnetic semiconductors like $\mathrm{ZnO}$ and try to get this phenomenon at higher temperatures. In the present work, we report on the magnetic properties of low Fe-doped $\mathrm{ZnO}$ nanowires (NWs) under the

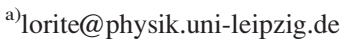

influence of light irradiation. Fe doping and the ionization of oxygen vacancies are found to be the key to observe PIM in nanowires up to $T \sim 270 \mathrm{~K}$. The PIM effect is observed only for Fe-doped $\mathrm{ZnO}(\mathrm{ZF})$ deficient in oxygen.

The ZF nanowires were prepared by a carbothermal process explained elsewhere. ${ }^{9,10}$ A mixture of $\mathrm{ZnO} / \mathrm{Fe}_{3} \mathrm{O}_{4} /$ carbon in the weight proportion of $30 \% / 20 \% / 50 \%$ was pressed applying $4 \mathrm{kPa}$ to form a pellet of $2 \mathrm{~mm}$ diameter. The pellet was kept in a furnace at $1150^{\circ} \mathrm{C}$ for $1 \mathrm{~h}$ and then cooled down to room temperature. A white foam-like powder formed by NWs was obtained on the wall at a distance of $15 \mathrm{~cm}$ from the center of tubular furnace.

The magnetic moment of a bundle of NWs was measured using a superconducting quantum interference device magnetometer (SQUID) from Quantum Design. The measurements of magnetization under illumination were performed with a Xe-lamp coupled to the SQUID. The light was guided by an optical fiber to the sample. The wavelength was selected by using the corresponding filters. The light intensity was kept low in order not to increase the sample temperature at the lowest measured temperatures.

Photoluminescence $(\mathrm{PL})$ of the NWs at room temperature was excited by the $325 \mathrm{~nm}$ line of a He-Cd KIMMON IK3301R-G laser operating in continuous-wave mode, spectrally dispersed by a $320 \mathrm{~mm}$ HORIBA JOBIN YVON iHR320 monochromator and detected by a Peltier-cooled CCD camera. X-ray diffraction measurements were performed using Philips Xpert diffractometer and Raman spectroscopy at room temperature was performed with a micro-Raman Jobin Yvon U1000 double spectrometer with excitation light of $532 \mathrm{~nm}$ and with a liquid nitrogen cooled CCD detector. Electronic paramagnetic resonance (EPR) measurements were carried out with a BRUKER EMX Micro X-band spectrometer at 9.41 GHz. The Q-band cw EPR spectra were recorded with a 

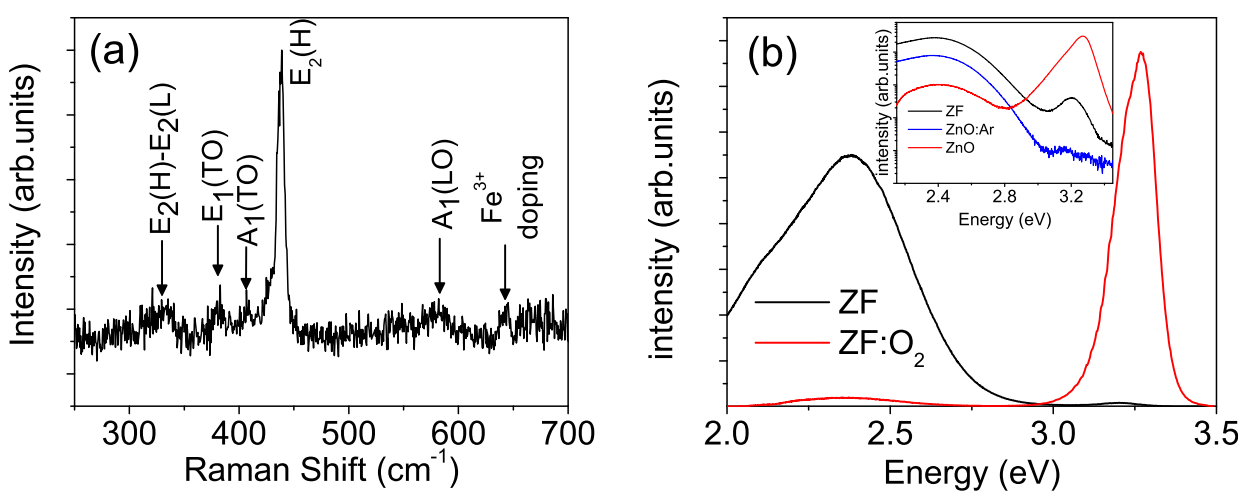

FIG. 1. (a) Raman spectroscopy of a bundle of ZF nanowires. (b) Photoluminescence of ZF NWs as prepared (in black) and after an annealing treatment at $400{ }^{\circ} \mathrm{C}$ in oxygen atmosphere (in red). The inset shows the photoluminescence of undoped $\mathrm{ZnO}$ NWs before (in red) and after $\mathrm{Ar}$ annealing at $400{ }^{\circ} \mathrm{C}$ (in blue), to produce oxygen vacancies, in comparison to ZF NWs (in black).
BRUKER EMX 10-40 spectrometer operating at $34 \mathrm{GHz}$. EPR spectra were simulated using the Easy Spin Matlab toolbox. $^{11}$

Scanning electron microscopy (SEM) measurements revealed that the synthesized wires have a diameter of $\leq 130 \mathrm{~nm}$ and several micrometers length. The XRD pattern of a bundle of ZF NWs along with that of undoped $\mathrm{ZnO}$. The XRD results indicate the existence of diffraction peaks of hexagonal $\mathrm{ZnO}$ as a single phase without the presence of secondary phases or Fe clusters, within experimental resolution. Particle induced x-ray emission (PIXE) measurements were carried out to quantify the amount of Fe in the NWs. We found a $\mathrm{Fe}$ concentration of $0.2 \pm 0.05$ at. \%. This Fe concentration is slightly smaller that the previously reported Fesolubility limit in $\mathrm{ZnO}^{9}$

Raman spectroscopy allows the observation of small amount of impurities, extra phases, or dopant in $\mathrm{ZnO} .{ }^{12,13} \mathrm{In}$ agreement with the XRD results, signatures of secondary phases have not been found in the Raman spectrum of the studied NWs (see Fig. 1), which shows the typical modes observed for $\mathrm{ZnO}{ }^{12}$ Note that the longitudinal vibrational mode $\mathrm{A}_{1}(\mathrm{LO})$ at $586 \mathrm{~cm}^{-1}$ is related to oxygen vacancies $\mathrm{V}_{\mathrm{O}}$. An additional feature, observed at $644 \mathrm{~cm}^{-1}$, has been previously related to the incorporation of $\mathrm{Fe}^{3+}$ ions at $\mathrm{Zn}$ sites. $^{12}$

The existence of $\mathrm{V}_{\mathrm{O}}$ was corroborated by PL measurements. Figure 1(b) shows the PL spectrum of the as grown Fe-doped ZnO NWs, which presents two different bands, i.e., a band edge emission and a defect level emission. The band edge emission at an energy $\simeq 3.2 \mathrm{eV}$ (wavelength $\lambda \simeq$ $388 \mathrm{~nm}$ ) is attributed to the recombination of free excitons and their phonon replica. ${ }^{14}$ The broad band at $2 \ldots 2.75 \mathrm{eV}$ $(600 \ldots 450 \mathrm{~nm})$ represents the defect level emission or green luminescence, which is commonly related to different type of lattice defects in the $\mathrm{ZnO}$ lattice. ${ }^{9}$ After an annealing at $400{ }^{\circ} \mathrm{C}$ for the oxygenation of the NWs, the observed green luminescence intensity is drastically reduced (see Fig. 1(b)). It suggests that it is mostly produced by $\mathrm{V}_{\mathrm{O}}$ 's. These vacancies are removed by the oxygen incorporation during the annealing treatment. It is important to mention that most of the $\mathrm{V}_{\mathrm{O}}$ 's in $\mathrm{ZnO}$ NWs are defects localized at the near surface region. The concentration of $\mathrm{V}_{\mathrm{O}}$ 's decreases strongly to the interior of NWs, ${ }^{15}$ and this facilitates its removal with low-temperature annealing treatments in oxygen atmosphere.

The change in the magnetic moment of the samples with light has been measured in Fe-doped and undoped $\mathrm{ZnO}$
NWs, before and after annealing in $\mathrm{O}_{2}$ atmosphere. Figures 2(a) and 2(b) shows the field hysteresis of the as prepared ZF NWs, in dark and in light; all samples of $6 \mathrm{mg}$ mass. The data here presented are after subtraction of a linear in field background. This background is due to the intrinsic diamagnetic contribution of $\mathrm{ZnO}$ and the sample holder where the samples were placed and a small paramagnetic (PM) contribution. Assuming a Curie-law temperature dependence for the PM contribution and a temperature independent diamagnetic one, we estimate a PM contribution of the order of $\sim 8 \%$ of the total Fe concentration in the ZF NWs, assuming $1 \mu_{B}$ per $\mathrm{Fe}$ ion.

In dark conditions, all the studied ZF NWs exhibit ferromagnetic ordering with a magnetic moment at saturation of $3.1 \times 10^{-4}$ emu and $7.9 \times 10^{-4}$ emu at $5 \mathrm{~K}$ and $300 \mathrm{~K}$, respectively (see Figs. 2(a) and 2(b)). The measured magnetic moment at saturation would correspond to $\sim 0.6 \mu_{B} / \mathrm{Fe}$ (at $5 \mathrm{~K}$ ), a value smaller than the value reported in literature. ${ }^{16}$
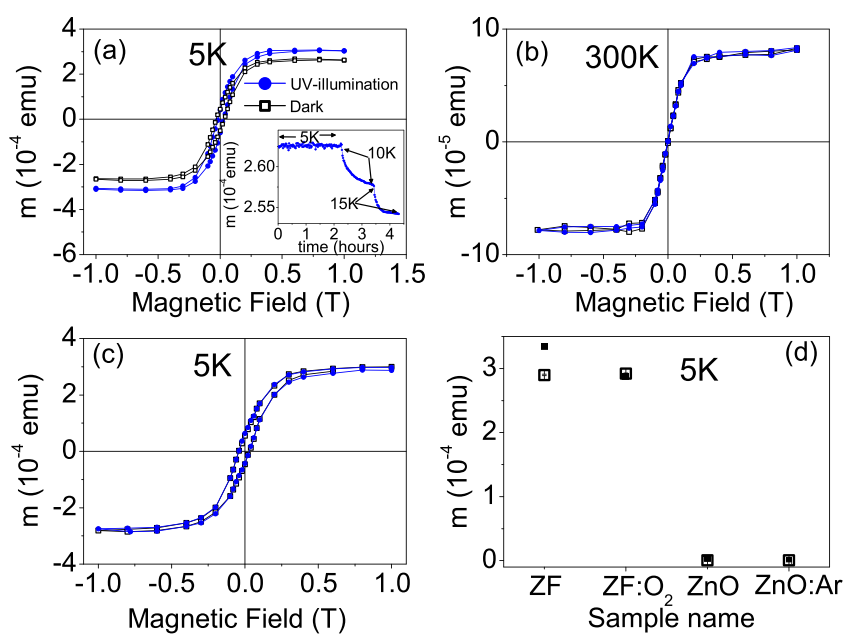

FIG. 2. Magnetic moment vs. applied magnetic field of $6 \mathrm{mg}$ of ZF NWs at (a) $5 \mathrm{~K}$ and (b) $300 \mathrm{~K}$ in dark (black squares) and under light (blue circles) at $\lambda=425 \mathrm{~nm}$. (c) Magnetic moment vs. applied magnetic field of $6 \mathrm{mg} \mathrm{ZF}$ NWs in dark and under illumination (same symbols as in (a) and (b)) at $\lambda=425 \mathrm{~nm}$ after annealing in oxygen, ZF:O. (d) Magnetic moment at saturation of $6 \mathrm{mg}$ NWs in dark (opened squares) and under illumination (black squares) for $\mathrm{ZF}$; $\mathrm{ZF}: \mathrm{O}_{2}$; undoped $\mathrm{ZnO}$ and undoped $\mathrm{ZnO}$ after annealing in $\mathrm{Ar}$ to increase the amount of O-vacancies, $\mathrm{ZnO}$ :Ar. The annealing treatments to obtain $\mathrm{ZF}: \mathrm{O}_{2}$ and $\mathrm{ZnO}$ :Ar were performed in the previously measured $\mathrm{ZF}$ and $\mathrm{ZnO}$, respectively. The inset in (a) shows the time independence of the PIM (after turning off the light) at $5 \mathrm{~K}$ and its exponential decrease in time after increasing the temperature. The applied field was $0.1 \mathrm{~T}$ in this measurement. 
Note that the Curie temperature $T_{C}>300 \mathrm{~K}$, in spite of the very low concentration of Fe. This fact suggests that regions should exist with a $\mathrm{Fe}$ concentration larger than the average 0.2 at. $\%$ to trigger magnetic order above room temperature, likely due to double exchange. The estimated low magnetic moment of $\mathrm{Fe}$ would also indicate that in addition to the FM contribution, $\mathrm{Fe}$ ions can also couple antiferromagnetically (AFM) by super-exchange interaction, ${ }^{16,17}$ in addition to the paramagnetic contribution. Taking into account the value of $1 \mu_{B}$ per Fe for double exchange couple pairs, ${ }^{16,17}$ we estimate that $\sim 30 \%$ of the $\mathrm{Fe}$ concentration is responsible for the FM signal, whereas most of the remaining $\mathrm{Fe}$ ions should be AFM ordered.

After measuring the magnetic moment of the asprepared ZF NWs in dark, we illuminate them with light of wavelength $\lambda=425 \mathrm{~nm}$. Figure 2(a) shows the observed increase in the magnetic moment of $\simeq 15 \%$ at saturation under light and at $5 \mathrm{~K}$. At this low temperature, the increase in the magnetic moment with light has a persistent character after switching the light off. This persistent light-driven enhancement of the ferromagnetic moment decreases with temperature, see inset in Figure 2(a). The same set of experiments was performed at different $\lambda=360 \mathrm{~nm}, 545 \mathrm{~nm}$, and $690 \mathrm{~nm}$; however, no change in the magnetization was detected within a relative resolution of $0.1 \%$, indicating that a specific range of wavelength is needed to obtain the PIM effect.

As shown previously, ZF NWs exhibit a green emission (see Fig. 1(b)), mostly due to the existence of $\mathrm{V}_{\mathrm{O}}$ 's in the asprepared NWs. To check for their role in the observed PIM, we explored the effect in the ZF NWs after annealing them in oxygen, see Fig. 1(b), removing in this way a large part of the $V_{O}$ 's. After reducing the oxygen vacancies content we measured the same magnetic moment as in the as-prepared NWs, in dark but also under illumination. This result indicates that the $\mathrm{V}_{\mathrm{O}}$ 's are important only for the PIM effect. Finally, to clarify the importance of Fe doping, similar experiments were performed in non-magnetic, pure $\mathrm{ZnO}$ asprepared as well as after Ar annealing to produce $\mathrm{V}_{\mathrm{O}}$ 's. This was corroborated by photoluminescence by the observation of the increase in the green band, in comparison to untreated pure $\mathrm{ZnO}$, with an intensity comparable to the one observed in ZF NWs (see inset in Fig. 1(b)). Ferromagnetic order in the $\mathrm{ZnO} \mathrm{NWs}$, independently of their $\mathrm{V}_{\mathrm{O}}$ 's concentrations, was observed neither in dark conditions nor under illumination. All these results indicate that both, $\mathrm{Fe}$ and $\mathrm{V}_{\mathrm{O}}$ 's are necessary to observe the PIM effect in $\mathrm{ZnO}$.

We measured the temperature dependence of the enhanced magnetization under constant UV illumination. The results are shown in Fig. 3(a). We observe that the enhancement, given by the difference between the results with and without light, decreases linearly with temperature above $50 \mathrm{~K}$, see Fig. 3(b), vanishing at $\approx 270 \mathrm{~K}$. This behavior can be an indication of the existence of spin waves in two dimensions (2D), as previously observed in defect-induced magnetic graphite after proton irradiation. ${ }^{18}$ This apparent 2D dimensionality of the PIM effect in our ZF NWs agrees with the expectation that the near surface region is the one where the PIM effect originates.
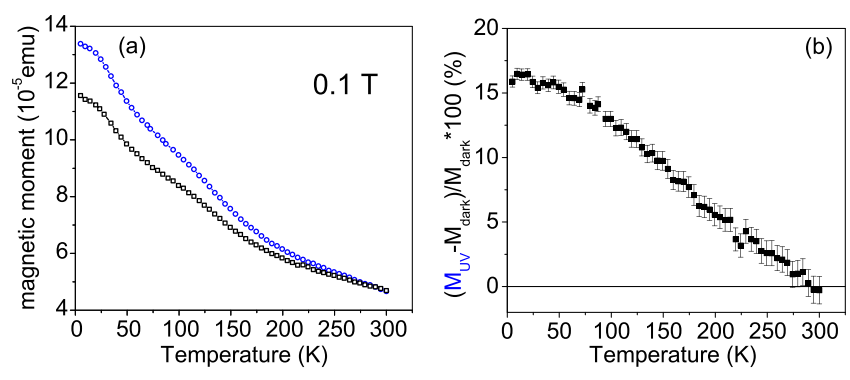

FIG. 3. (a) Temperature dependence of ZF magnetization in dark condition (black points) and during illumination and (b) temperature dependence of the photo induced enhancement of the magnetization.

To further clarify the mechanisms behind the magnetic order and the PIM origin observed in the ZF NWs, electronic paramagnetic resonance (EPR) measurements were performed between $7 \mathrm{~K}$ and $503 \mathrm{~K}$. In Fig. 4, EPR measurements of a bundle of ZF NWs at X- and Q-band frequencies are presented. At room temperature, the $\mathrm{X}$-band measurements show a broad signal around $g=2$. Attributing these signals to $\mathrm{Fe}^{3+}$ ions we suggest that $\mathrm{Fe}^{3+}$ is not magnetically diluted and magnetic dipolar and exchange interactions between $\mathrm{Fe}$-ions lead to a coalescence of the fine structure pattern of $\mathrm{Fe}^{3+}$. The signal can be simulated by two main species, A and B, see inset in Fig. 4(a), which are observed in a wide temperature range. The X-band measurements show an apparent $g$-value increase from 2.03 at $503 \mathrm{~K}$ to about 2.35 at $7 \mathrm{~K}$. To study the nature of the EPR signals, Q-band measurements were conducted (see Fig. 4(b)). Here, we observe a slight increase in the $g$-factor from 2.035 at room temperature to 2.06 at $15 \mathrm{~K}$, suggesting the presence of temperature dependent internal fields which can be estimated from the comparison of the Xand Q-band spectra ${ }^{19}$ and are shown in the inset of Fig. 4(b). This allows us to recalculate the real value of $g=2.00$ (1) at room temperature, which is in a good agreement with the value for $\mathrm{Fe}^{3+} \cdot{ }^{19}$ Additionally, we observe that the line width increases towards lower temperatures, see Fig. 4(a), suggesting that the Fe ions observed by EPR do not couple only ferromagnetically ${ }^{20}$ but an antiferromagnetic coupling exists. With the presence of internal fields attributed to Fe-ions ferromagnetically coupled, the overall EPR results support the interpretation of the magnetization that both FM and AFM ordered Fe-ions co-exist in the studied NWs. ${ }^{21}$
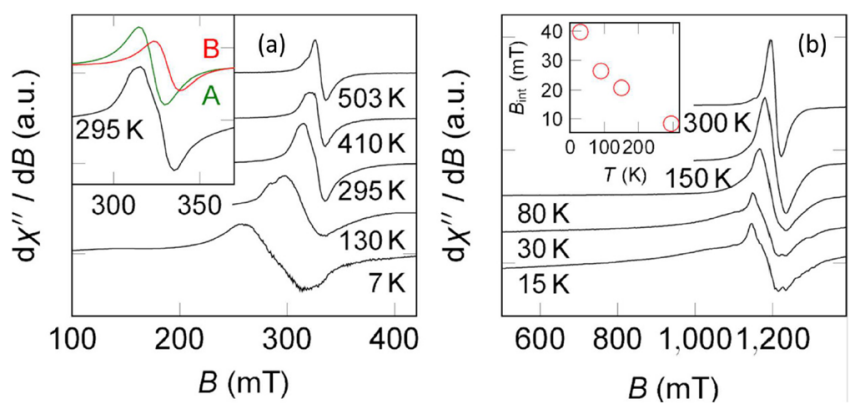

FIG. 4. (a) Experimental EPR spectra at X-band frequency of ZF NWs at temperatures from $7 \mathrm{~K}$ to $503 \mathrm{~K}$. The inset shows the two components of the room temperature signal. (b) Q-band EPR spectra of ZF nanowires at temperatures between $15 \mathrm{~K}$ and room temperature. The inset shows the calculated internal fields for species A. 
As noted above, to explain the observed magnetic order at such high temperatures and with such small $\mathrm{Fe}$ concentration, we need to assume the formation of a non homogeneous distribution of Fe-ions which allows the co-existence of FM, $\mathrm{Fe}^{2+}-\mathrm{Fe}^{3+}$, and AFM regions, $\mathrm{Fe}^{3+}-\mathrm{Fe}^{3+}$. Moreover, the $\mathrm{V}_{\mathrm{O}}$ 's can be placed in the neighborhood of an AFM couple. By light illumination, the aforementioned $\mathrm{V}_{\mathrm{O}}$ can be ionized to form $\mathrm{V}_{O^{+}}$. When the $\mathrm{V}_{\mathrm{O}}$ is close to an $\mathrm{Fe}^{3+}$, this photoionization can produce an electron transfer from the $\mathrm{V}_{\mathrm{O}}$ to the nearby $\mathrm{Fe}^{3+}$ ion transforming it into $\mathrm{Fe}^{2+}$. This will produce $\mathrm{Fe}^{2+}-\mathrm{Fe}^{3+} \mathrm{FM}$ couple pairs increasing the density of double exchange couples that contribute to the FM order in detriment of the pairs AFM ordered.

The found PIM effect in $\mathrm{ZnO}$ doped with a nominally low concentration of Fe and our interpretation based on the light-induced ionization of the oxygen vacancies near $\mathrm{Fe}$ ions, localized mainly at the surface of the NWs, shed light to the possibility of exploring light-induced magnetism in semiconductors at much higher temperatures than the ones reported previously. The effect found in our study could be applied to other types of oxides in which the ionization of vacancies by light is possible. The PIM effect and the possibility to have it in nanostructures as small or smaller than in our NWs should substantially support further development of magnetic semiconductors for optospintronics application at technologically relevant wavelengths.

This work was partially supported by CIUNT under Grant Nos. 26/E439 and 26/E478, by ANPCyT-PICTR 35682, and by the Collaborative Research Center SFB 762 "Functionality of Oxide Interfaces." We are grateful for the support within the DFG priority program SPP 1601 "New Frontiers in Sensitivity for EPR Spectroscopy."
${ }^{1}$ O. Sato, T. Iyoda, A. Fujishima, and K. Hashimoto, Science 272, 704 (1996).

${ }^{2}$ S. Koshihara, A. Oiwa, M. Hirasawa, S. Katsumoto, Y. Iye, C. Urano, H. Takagi, and H. Munekata, Phys. Rev. Lett. 78, 4617 (1997).

${ }^{3}$ A. Pandey, S. Brovelli, R. Viswanatha, L. Li, J. M. Pietryga, V. I. Klimov, and S. A. Crooker, Nat. Nanotechnol. 7, 792 (2012).

${ }^{4}$ W. D. Rice, P. Ambwani, M. Bombeck, J. D. Thompson, G. Haugstad, C. Leighton, and S. A. Crooker, Nat. Mater. 13, 481 (2014).

${ }^{5}$ J. S. Bettinger, R. V. Chopdekar, and Y. Suzuki1, Appl. Phys. Lett. 94, 072505 (2009).

${ }^{6}$ M. Althammer, E.-M. Karrer-Müller, S. T. B. Goennenwein, M. Opel, and R. Gross, Appl. Phys. Lett. 101, 082404 (2012).

${ }^{7}$ I. Lorite, Y. Kumar, P. Esquinazi, C. Zandalazini, and S. P. de Heluani, Small 11, 4403 (2015).

${ }^{8}$ S. T. Ochsenbein, Y. Feng, K. M. Whitaker, E. Badaeva, W. K. Liu, X. Li, and D. R. Gamelin, Nature Nanotechnology 4, 681 (2009).

${ }^{9}$ B. Aleman, Y. Ortega, J. A. García, P. Fernandez, and J. Piqueras, J. Appl. Phys. 110, 014317 (2011).

${ }^{10}$ I. Lorite, P. Esquinazi, C. Zapata, and S. P. Heluani, J. Mater. Res. 29, 78 (2014).

${ }^{11}$ S. Stoll and A. Schweiger, J. Magn. Reson. 178, 42 (2006).

${ }^{12}$ C. Bundesmann, N. Ashkenov, M. Schubert, D. Spemann, T. Butz, E. M. Kaidashev, M. Lorenz, and M. Grundmann, Appl. Phys. Lett. 83, 1974 (2003).

${ }^{13}$ I. Lorite, F. Rubio-Marcos, J. Romero, and J. Fernández, Mater. Lett. 63, 212 (2009).

${ }^{14}$ W. Shan, W. Walukiewicz, J. W. Ager, K. M. Yu, H. B. Yuan, H. P. Xin, G. Cantwell, and J. J. Song, Appl. Phys. Lett. 86, 191911 (2005).

${ }^{15}$ K. M. Wong, S. M. A. e Abbas, Y. Fang, A. Shaukat, and Y. Lei, J. Appl. Phys. 114, 034901 (2013).

${ }^{16}$ D. Karmakar, S. K. Mandal, R. M. Kadam, P. L. Paulose, A. K. Rajarajan, T. K. Nath, A. K. Das, I. Dasgupta, and G. P. Das, Phys. Rev. B 75, 144404 (2007).

${ }^{17}$ R. H. Kodama and A. E. Berkowitz, Phys. Rev. B 59, 6321 (1999).

${ }^{18}$ J. Barzola-Quiquia, P. Esquinazi, M. Rothermel, D. Spemann, T. Butz, and N. García, Phys. Rev. B 76, 161403(R) (2007).

${ }^{19}$ A. Fainstein, E. Winkler, A. Butera, and J. Tallon, Phys. Rev. B 60, R12597 (1999).

${ }^{20}$ B. Schulz, R. Schwarzwald, and K. Baberschke, Surf. Sci. 307-309, 1102 (1994).

${ }^{21}$ H. de Wijn, L. Walker, J. L. Davis, and H. Guggenheim, Solid State Commun. 11, 803 (1972). 